\title{
A New and Useful Syntactic Restriction on Rule Semantics for Tabular Data
}

\author{
Marie Agier ${ }^{1,2}$, Jean-Marc Petit ${ }^{3}$ \\ ${ }^{1}$ DIAGNOGENE SA, 15000 Aurillac, FRANCE \\ ${ }^{2}$ LIMOS, UMR 6158 CNRS, Univ. Clermont-Ferrand II, FRANCE \\ ${ }^{3}$ LIRIS, UMR 5205 CNRS, INSA Lyon, FRANCE
}

\begin{abstract}
Different rule semantics have been defined successively in many contexts such as functional dependencies in databases or association rules in data mining to mention a few. In this paper, we focus on the class of rule semantics for tabular data for which Armstrong's axiom system is sound and complete, so-called well-formed semantics. The main contribution of this paper is to show that an equivalence does exist between some syntactic restrictions on the natural definition of a given semantics and the fact that this semantics is well-formed. From a practical point of view, this equivalence allows to prove easily whether or not a new semantics is well-formed. Moreover, the same reasoning on rules can be performed over any well-formed semantics.

We also point out the relationship between our generic definition of rule satisfaction and the underlying data mining problem, i.e. given a well-formed semantics and
\end{abstract}

a relation, discover a cover of rules satisfied in this relation.

This work takes its roots from a bioinformatics application, the discovery of gene regulatory networks from gene expression data.

Keywords Rules, implications, Armstrong's axiom system, data mining.

\section{Introduction}

The notion of rules or implications is very popular and appears in different flavors in databases, data mining or artificial intelligence communities. The two more famous examples of rules are association rules [5] and functional dependencies [7]. As such, a simple remark can be done on such rules: their syntax is the same but their semantics widely differs. In this paper, we consider rules to be defined on tabular datasets. Basically, tabular dataset is equivalent to a relation over a set $U$ of distinguished at- 
tributes (or columns) in databases terminology [1]. In this context, a rule is an expression of the shape $X \rightarrow Y$ i.e. "X implies Y" with $X, Y \subseteq U$.

The semantics of a rule $X \rightarrow Y$ over $U$ is the meaning, the sense one wants to give to this rule: Given a relation $r$, a rule $X \rightarrow Y$ is said to be satisfied in $r$ if the semantics of the rule is true (or valid) in $r$. We identified three main components to specify a semantics for rules in a relation: The type of the data, the subsets of the relation on which the rule applies and the predicates occurring in the "if... then..." part of the rule. We proposed in this paper a natural and "generic" definition of a semantics in order to be able to capture most of existing semantics already known on tabular data.

Note that error measures and quality measures are not taken into account in our generic definition of a semantics. These measures can be generally applied to many different semantics and do not belong to what we believe to be the core definition of a rule semantics. Moreover, we do not want to define as many semantics as there are measures. In a data mining context, error and quality measures can be integrated a posteriori to sort and to qualify the rules.

Furthermore, we focus on those semantics verifying Armstrong's axioms, so-called "well-formed semantics" [4], i.e. semantics for rules on which Armstrong's axiom system applies. The practical interests are twofold:
- Firstly, reasoning can be performed on rules from the Armstrong's axioms. For instance, given a set of rules $F$, it is possible to know if a rule is implied by this set of rules in linear time [11].

- It is also possible to work on "small" covers of rules $[27,19,18]$ and to use a discovery process specific to the considered cover, but applicable to all wellformed semantics.

Paper contribution The contribution of this paper is to show that an equivalence does exist between some syntactic restrictions on the natural definition of a given semantics and the fact that this semantics is well-formed.

From a practical point of view, this equivalence allows to prove easily that a new semantics is well-formed: So far, for a given semantics, we had to give a proof of the soundness and the completeness of the Armstrong's axiom system for this semantics, this proof being not always trivial. Now, it is just enough to show that this semantics complies with the proposed syntactic restrictions.

We also point out the relationship between our generic definition of rule satisfaction and the underlying data mining problem, i.e. given a well-formed semantics and a relation, discover a cover of rules satisfied in this relation. More precisely, we show how a base of the closure system for any well-formed semantics can be computed from the dataset. 
Application This work takes its roots from a bioinformatics application, the discovery of gene regulatory networks from gene expression data. The challenge is to find out relationships between genes that reflect observations of how expression level of each gene affects those of others. The conjecture that association rules could be a model for the discovery of gene regulatory networks has been partially validated in $[10,21,14,13]$. Nevertheless, we believe that many different kinds of rules between genes could be useful with respect to some biological objectives and the restricted setting of association rules could be not enough to cope with this diversity. Three different types of rules were already proposed in [3] for gene expression data.

In this context, the main application of this paper is to offer a framework in which biologists may define their "own customized semantics" for rules with regard to their requirements. Once a semantics is proved well-formed, i.e. this semantics just complies with the proposed syntactic restrictions, well-known inference methods on rules can be performed for biologists.

This work has been intended for gene expression data, however, in this paper we extend our proposition to all types of tabular data.

Paper organization We give in Section 2 some examples of rule semantics. In Section 3, we propose a natural definition of a semantics using some syntactic restric- tions. In Section 4, we further restrict the syntax and give the main result of this paper. We point out in Section 5 some relationships between our proposition and the underlying data mining problem. In Section 6 , we give the related contributions of this work and finally, we conclude and give some perspectives in Section 7.

\section{Motivating examples}

To show the interest of our proposition, we give in the sequel four examples of semantics for tabular data, some of them in the context of gene expression data [3]. These examples show that many rule semantics can be defined.

Let $r$ be a relation over $U$ and $X, Y \in U$ two subsets of attributes. In the context of gene expression data, an attribute is a gene and the domain of attributes is the set of real numbers.

Example 1 Let $s_{1}$ be a semantics studying for example the levels of expression of genes, $s_{1}$ can be defined as follows, using two usersupplied thresholds $\varepsilon_{1}$ and $\varepsilon_{2}$ :

$r \models s_{1} X \rightarrow Y$ if and only if $\forall t \in r$, if $\forall A \in$ $X, \varepsilon_{1} \leq t[A] \leq \varepsilon_{2}$ then $\forall A \in Y, \varepsilon_{1} \leq t[A] \leq$ $\varepsilon_{2}$.

This semantics points out a semantics close to association rules without discretization phase.

Example 2 Let $s_{2}$ be a semantics studying for example the evolution of gene expression 
levels, $s_{2}$ can be defined as follows, using two user-supplied thresholds $\varepsilon_{1}$ and $\varepsilon_{2}$ : $r \models_{s_{2}} X \rightarrow Y$ if and only if $\forall t_{i}, t_{i+1} \in r$, if $\forall A \in X, \varepsilon_{1} \leq t_{i+1}[A]-t_{i}[A] \leq \varepsilon_{2}$ then $\forall A \in Y, \varepsilon_{1} \leq t_{i+1}[A]-t_{i}[A] \leq \varepsilon_{2}$.

Note that an order has to exist among tuples, such a constraint being implicitly expressed with indices on tuples.

Example 3 Let $s_{d}$ be a new semantics studying the Euclidian distance between for example gene expression profiles, $s_{d}$ can be defined as follows, using two user-supplied thresholds $\varepsilon_{1}$ and $\varepsilon_{2}$ :

$r \models_{s_{d}} X \rightarrow Y$ if and only if $\forall t_{i}, t_{j} \in r$, if $\varepsilon_{1} \leq d\left(t_{i}[X], t_{j}[X]\right) \leq \varepsilon_{2}$ then $\varepsilon_{1} \leq$ $d\left(t_{i}[Y], t_{j}[Y]\right) \leq \varepsilon_{2}$.

These various examples show that with the same syntax, a rule may have very different meanings and from the same dataset, several semantics can be defined and interesting for the experts.

\section{$3 \quad$ Well-formed semantics}

We identified three components in the definition of a semantics for rules in a relation:

- The type of the data: Rule semantics can be generally applied for some restricted types of data, for example binary or categorical attributes, temporal data, presence of classes... The nature of the data being analyzed clearly influences the definition of a semantics.
- The subsets of the relation on which a rule applies: An important characteristic of a semantics is the condition on tuples to take into account. We can for example study tuples one by one (like association rules), we can do a pairwise comparison of tuples (like functional dependencies) or compare the tuple $i$ with the tuple $i+1$ or the tuple $i$ with the tuples $j$ where $j>i \ldots$ The semantics widely differ depending on these characteristics.

- The predicates occurring in the "if Pred $_{1}$ is true then Pred 2 is true" part of the rule: Predicates Pred $_{1}$ and Pred $_{2}$ are defined on a set of attributes and a subset of the relation. Note that these two predicates can be the same. For example, for functional dependencies, the predicates can be formulated as: $\left[\forall A \in X, t_{1}[A]=t_{2}[A]\right]$, where $t_{1}, t_{2}$ are two tuples, and $X$ is a subset of attributes. These predicates really give the meaning of the semantics.

A "generic" definition of a semantics based on these three components, is described in the sequel.

\subsection{Generic definition of a seman- tics}

Given a relation $r$, the satisfaction of a rule $X \rightarrow Y$ in $r$ for a semantics $s$, noted $r \models_{s}$ $X \rightarrow Y$, can be defined in a general way as follows: 
Definition 1 Let $X, Y \subseteq U$ and $r$ a relation over $U$. The satisfaction of the rule $X \rightarrow Y$ in $r$ for a semantics $s$, noted $r \models_{s} X \rightarrow Y$, is defined by:

$r \models_{s} X \rightarrow Y$ if and only if $\forall r^{\prime} \subseteq r$ verifying $d_{c}\left(r^{\prime}\right)$, if $\operatorname{Pred}_{1}\left(X, r^{\prime}\right)$ is true then $\operatorname{Pred}_{2}\left(Y, r^{\prime}\right)$ is true where:

1. $d_{c}\left(r^{\prime}\right)$ specifies a constraint which has to be verified over $r^{\prime} \subseteq r$.

2. $\operatorname{Pred}_{1}\left(X, r^{\prime}\right)\left(\right.$ resp. $\left.\operatorname{Pred}_{2}\left(Y, r^{\prime}\right)\right)$ is a predicate specifying a condition on $X$ (resp. $Y$ ) over $r^{\prime}$.

A semantics is thus characterized by a constraint $d_{c}$ defined on a subset of tuples and by two predicates Pred 1 and Pred $_{2}$ defined for a subset of attributes.

Predicates are logical expressions returning true or false. They are defined on $X$ and $r^{\prime}$ only, no other attributes or subset of $r$ are allowed in this definition.

The formal description of these logical expressions is quite important while being somewhat tedious. For the time being, we prefer to give the intuition through examples. In future works, we plan to give the formal definition of $d_{c}$, Pred 1 and Pred $_{2}$.

Note that pathological predicates logically equivalent to true (or false) should be taken into account in our definition, but are useless in practice. In the sequel, such predicates will not be considered.

Example 4 The semantics $s_{1}$ presented in example 1 can be characterized by the con- straint $d_{c}$ and the predicate defined as follows:

1. $d_{c}\left(r^{\prime}\right)=\left[r^{\prime}=\{t\}\right.$ with $\left.t \in r\right]$.

2. $\operatorname{Pred}_{1}(X,\{t\})=\operatorname{Pred}_{2}(X,\{t\})=$ $\left[\forall A \in X, \varepsilon_{1} \leq t[A] \leq \varepsilon_{2}\right]$.

In the sequel, we shall say that a semantics $s$ complies with definition 1 if $s$ can be syntactically expressed within the setting of definition 1.

Moreover, we shall note by $C$ the class of rule semantics complying with definition 1 .

It is worth noting that we do not integrate quality or error measures of a rule in the "core" definition of a semantics. Roughly speaking, two types of measures can characterize rules, error measures and quality measures:

- Error measures like for example confidence defined for association rules or error indications defined for functional dependencies [22], allow to append approximate rules to exact rules, i.e. those rules which are almost satisfied. These measures are very interesting since they allow to take into account noise in data.

- Quality measures like support, dependency or informative rate [5, 30], allow at contrary to limit the number of rules and possibly to sort out the obtained rules. These measures allow to give to the experts the rules which seem to be 
the most surprising, the most "interesting" with regard to the chosen statistical criteria.

They can be integrated a posteriori to sort and to qualify the rules. Those error and quality measures will not be discussed anymore in the rest of this paper.

Example 5 The semantics $s_{2}$ belongs to $C$ since it can be characterized by the constraint $d_{c}$ and the predicate defined as follows:

1. $d_{c}\left(r^{\prime}\right)=\left[r^{\prime}=\left\{t_{i}, t_{i+1}\right\}\right.$ with $t_{i}, t_{i+1} \in$ $r]$.

2. $\operatorname{Pred}_{1}\left(X,\left\{t_{i}, t_{i+1}\right\}\right)=\operatorname{Pred}_{2}(X$, $\left.\left\{t_{i}, t_{i+1}\right\}\right)=\left[\forall A \in X, \varepsilon_{1} \leq\right.$ $\left.t_{i+1}[A]-t_{i}[A] \leq \varepsilon_{2}\right]$.

Example 6 The semantics $s_{d}$ belongs to $C$ since it can be characterized by the constraint $d_{c}$ and the predicate defined as follows:

1. $d_{c}\left(r^{\prime}\right)=\left[r^{\prime}=\left\{t_{i}, t_{j}\right\}\right.$ with $\left.t_{i}, t_{j} \in r\right]$.

2. $\operatorname{Pred}_{1}\left(X,\left\{t_{i}, t_{j}\right\}\right)=\operatorname{Pred}_{2}\left(X,\left\{t_{i}, t_{j}\right\}\right)$ $=\left[\varepsilon_{1} \leq d\left(t_{i}[X], t_{j}[X]\right) \leq \varepsilon_{2}\right]$.

To conclude, the definition 1 of a semantics has been devised as naturally as possible to capture a great variety of rules. Nevertheless, this definition does not accept everything as shown in the following example:

Example 7 Consider the semantics of inclusion dependencies defined as follows: $r \models R[X] \subseteq R[Y]$ if and only if $\forall t \in r, \exists t^{\prime} \in r$ such that $t[X]=t^{\prime}[Y]$.

Due to the test $t[X]=t^{\prime}[Y]$, a condition on $\mathrm{X}$ and Y's attributes has to be specified i.e. Pred $_{1}$ (or Pred 2 ) has to use X and Y in its definition. Therefore, the satisfaction of inclusion dependencies cannot be expressed within definition 1 , thus this semantics does not belong to $C$.

\subsection{Framework of well-formed se- mantics}

A general framework can be borrowed from theoretical investigations performed over functional dependencies and Armstrong's axiom system $[28,16]$. This framework allows to resume interesting properties defined for functional dependencies like reasoning on rules and generating covers for rules.

To be sure that a semantics fulfills this framework, the notion of well-formed semantics can be defined as follows:

Definition 2 A semantics $s$ is well-formed if Armstrong's axiom system is sound and complete for $s$.

Let us recall the Armstrong's axiom system for a set of rules $F$ defined over a set of attributes $U$ :

1. (reflexivity) if $X \subseteq Y \subseteq U$ then $F \vdash$ $Y \rightarrow X$ 
2. (augmentation) if $F \vdash X \rightarrow Y$ and $W \subseteq U$, then $F \vdash X W \rightarrow Y W$

3. (transitivity) if $F \vdash X \rightarrow Y$ and $F \vdash$ $Y \rightarrow Z$ then $F \vdash X \rightarrow Z$

The notation $F \vdash X \rightarrow Y$ means that a proof of $X \rightarrow Y$ can be obtained using Armstrong's axiom system from $F$. Moreover, given a semantics $s$, the notation $F \models{ }_{s} X \rightarrow Y$ means that for all relations $r$ over $U$, if $r \models_{s} F$ then $r \models_{s} X \rightarrow Y$.

In other words, for any well-formed semantics $s, \vdash$ and $\models_{s}$ coincide.

To know whether or not a given semantics is well-formed, we have to give a proof of the soundness and the completeness of the Armstrong's axiom system for this semantics.

This proof being not always trivial, the idea is to find further syntactic restrictions on rule satisfaction definition in order to ensure the well-formedness of the semantics.

\section{More syntactic restric- tions}

In this setting, we propose some syntactic restrictions on the definition 1 , which ensure that a semantics is well-formed. In other words, given a new semantics, we do not have to prove anything to be sure that Armstrong's axioms apply: It is just enough that the semantics complies with these syntactic restrictions.
Definition 3 Let $X, Y \subseteq U$ and $r$ a relation over $U$. The satisfaction of the rule $X \rightarrow Y$ in $r$ for a semantics $s$, noted $r \models{ }_{s} X \rightarrow Y$, is defined by:

$r \models_{s} X \rightarrow Y$ if and only if $\forall r^{\prime} \subseteq r$ verifying $d_{c}\left(r^{\prime}\right)$, if $\forall A \in X, \operatorname{Pred}\left(A, r^{\prime}\right)$ is true then $\forall A \in Y, \operatorname{Pred}\left(A, r^{\prime}\right)$ is true where:

1. $d_{c}\left(r^{\prime}\right)$ specifies a constraint which has to be verified over $r^{\prime} \subseteq r$.

2. $\operatorname{Pred}\left(A, r^{\prime}\right)$ is a predicate specifying a condition on $A$ over $r^{\prime}$.

The first item of this new semantic definition does not change with regard to the definition 1. Nevertheless, the difference is twofold:

- Firstly, the two predicates Pred $_{1}$ and Pred $_{2}$ are equivalent.

- Secondly, a restriction is posed on the predicate: Now, it must be satisfied for each single attribute $A \in X$ instead of being satisfied for the subset of attributes $X$.

Since two syntactically different definitions of predicates could be equivalent, we need to precisely define what "equivalent" means. Intuitively, two predicates are equivalent if for any relation $r$ and for any subset of attributes $X \subseteq U$, Pred $_{1}$ and $\mathrm{Pred}_{2}$ are satisfied for $X$ on the same subsets of $r$. Here is the formal definition:

Definition 4 Two predicates Pred $_{1}$ and Pred $_{2}$ are said to be equivalent, denoted by $\operatorname{Pred}_{1} \equiv \operatorname{Pred}_{2}$, if and only if for any 
relation $r$ and for any subset of attributes $X \subseteq U$, we have:

$\left\{r^{\prime} \subseteq r \mid r^{\prime}\right.$ verifying $d_{c}\left(r^{\prime}\right)$ and $\operatorname{Pred}_{1}\left(X, r^{\prime}\right)$ is true $\}=\left\{r^{\prime} \subseteq r \mid r^{\prime}\right.$ verifying $d_{c}\left(r^{\prime}\right)$ and $\operatorname{Pred}_{2}\left(X, r^{\prime}\right)$ is true $\}$.

In the sequel, we shall note by $C_{A}$ the class of rule semantics complying with definition $3, C_{A}$ being a subset of $C$.

Recall that (trivial) semantics build from predicates logically equivalent to true (or false) are not considered in $C$.

\subsection{Main result}

The main result of the paper gives an equivalence between well-formed semantics and semantics complying with definition 3 and is stated as follows:

Theorem 1 Let $s \in C$ be a rule semantics. The semantics $s$ is well-formed if and only if $s \in C_{A}$.

Proof 1 The proof is given in Appendix.

This theorem shows that among semantics complying with definition 1 , only those complying with definition 3 are well-formed and any well-formed semantics can be expressed within the syntactic restrictions given in definition 3 .

\subsection{Usefulness of these syntactic restrictions}

We shall point out the interest of this result through several examples.
Example 8 The semantics $s_{1}$ (see example 4), can be characterized by the constraint $d_{c}$ and the predicate Pred defined as follows:

1. $d_{c}\left(r^{\prime}\right)=\left[r^{\prime}=\{t\}\right.$ with $\left.t \in r\right]$.

2. $\operatorname{Pred}(A,\{t\})=\left[\varepsilon_{1} \leq t[A] \leq \varepsilon_{2}\right]$.

Thus, the semantics $s_{1} \in C_{A}$ and this is sufficient from Theorem 1, to prove the following result:

Corollary 1 The semantics $s_{1}$ is wellformed.

Example 9 The semantics $s_{2}$ (see example 5), can be characterized by the constraint $d_{c}$ and the predicate Pred defined as follows:

1. $d_{c}\left(r^{\prime}\right)=\left[r^{\prime}=\left\{t_{i}, t_{i+1}\right\}\right.$ with $t_{i}, t_{i+1} \in$ $r]$.

2. $\operatorname{Pred}\left(A,\left\{t_{i}, t_{i+1}\right\}\right)=\left[\varepsilon_{1} \leq t_{i+1}[A]-\right.$ $\left.t_{i}[A] \leq \varepsilon_{2}\right]$.

Thus, the semantics $s_{2} \in C_{A}$ and this is sufficient from Theorem 1, to prove the following result:

Corollary 2 The semantics $s_{2}$ is wellformed.

Example 10 For the semantics $s_{d}$ (see example 6), we have the following result:

Corollary 3 The semantics $s_{d}$ is not wellformed. 
Proof 2 We have to show that the semantics $s_{d}$ does not comply with definition 3. The semantics $s_{d}$ complies with definition 1, but the predicate $\operatorname{Pred}\left(X,\left\{t_{i}, t_{j}\right\}\right)$ $=\left[\varepsilon_{1} \leq d\left(t_{i}[X], t_{j}[X]\right) \leq \varepsilon_{2}\right]$, for $X \subseteq$ $U$ is obviously different from the predicate $\operatorname{Pred}^{\prime}\left(X,\left\{t_{i}, t_{j}\right\}\right)=\left[\forall A \in X, \varepsilon_{1} \leq\right.$ $\left.d\left(t_{i}[A], t_{j}[A]\right) \leq \varepsilon_{2}\right]$. It is easy to give a counter example where $\operatorname{Pred}(X, r)$ is true and $\operatorname{Pred}^{\prime}(X, r)$ is false. Thus, $s_{d} \notin C_{A}$ and by Theorem 1 , the result follows.

\section{Some relationships with data mining}

In a KDD context, the discovery of rules in tabular data has mainly been studied in the context of association rules for binary data [5] and functional dependencies [16, 20, 25]. In the setting of this paper, we may question about the underlying discovery problem for any well-formed semantics.

We are only interested in the context of this paper of semantics $s \in C_{A}$ i.e. the well-formed semantics. Rule generation for a semantics $s \in C \backslash C_{A}$ is also interesting but out of the scope of this paper.

First, recall that a one-to-one correspondence does exist between a set of rules and a closure system ${ }^{1}$ [17]. Second, given a relation $r$ and a well-formed semantics $s$, we can

\footnotetext{
${ }^{1}$ A closure system $C$ on $U$ is such that $U \in C$ and $\forall X, Y \in C, X \cap Y \in C$.
}

always define a closure system with respect to the set of satisfied rules in the relation $r$ for the semantics $s$.

Two main techniques do exist to compute a cover of rules:

- Those which enumerate the closure system to generate for example a minimum cover [19], generally used for association rules generation [31].

- Those which avoid the enumeration of the closure system to generate the canonical cover, generally used for the inference of functional dependencies $[28,16]$.

In both cases, the first step is to compute a $b_{a s e^{2}}$ of the closure system in the dataset, data accesses being made only during this step. This step is obviously specific to the considered semantics.

In this paper, we show how a base of the closure system for any well-formed semantics can be computed from the dataset.

First, we define a base with regard to our generic definition of rule semantics and then we give a proof that this is indeed a base:

Definition 5 Let $r$ a relation over $U$ and $s$ a given well-formed semantics. Let $B_{s}(r)$ the set defined as follows:

$$
\begin{gathered}
B_{s}(r)= \\
\bigcup_{r^{\prime} \subseteq r \mid d_{c}\left(r^{\prime}\right)}\left\{A \in U \mid \operatorname{Pred}\left(A, r^{\prime}\right) \text { is true }\right\} .
\end{gathered}
$$

\footnotetext{
${ }^{2}$ Also called agree sets for functional dependencies.
} 
We have the following result which extends in our context a well-known result obtained in the setting of functional dependencies [12]:

Proposition $1 B_{s}(r)$ is a base of the closure system with respect to the set $F_{s}(r)$ of satisfied rules in $r$ for the semantics $s$.

Proof 3 Let us recall that a sub-family $B$ of a closure system $C$ is a base if Inf $\subseteq B \subseteq$ $C$ where Inf is the set of meet-irreducible sets.

We first prove that $B_{s}(r) \subseteq C\left(F_{s}(r)\right)$ and then that $\operatorname{Inf}_{s}(r) \subseteq B_{s}(r)$, where $\operatorname{Inf_{s}}(r)$ is the set of meet-irreducible sets of the closure system $C\left(F_{s}(r)\right)$.

Let $X \in B_{s}(r)$, we have to prove that $X \in C\left(F_{s}(r)\right)$ i.e. that $X=X^{+}$. Suppose to the contrary that $\exists A \in U \backslash X$ such that $r \models_{s} X \rightarrow A$. In that case, $\forall r^{\prime} \subseteq r$ verifying $d_{c}\left(r^{\prime}\right)$ such that $\forall B \in X, \operatorname{Pred}\left(B, r^{\prime}\right)$ is true, we must have $\operatorname{Pred}\left(A, r^{\prime}\right)$ is true. That leads to a contradiction, since $X \in$ $B_{s}(r)$.

Let $X \in I n f_{s}(r)$, we have to prove that $X \in B_{s}(r)$. By definition, $X=X^{+}$and thus, for any $A \in U \backslash X, r \nvdash_{s} X \rightarrow A$ i.e. for any $A \in U \backslash X, \exists Y_{A} \in B_{s}(r)$ such that $X \subseteq Y_{A}$ and $A \notin Y_{A}$. As above, $Y_{A} \in$ $C\left(F_{s}(r)\right)$ for all $A \in U \backslash X$. Now, we have: $X=\bigcap_{A \in U \backslash X}\left(Y_{A}\right)$ and since $X \in I n f_{s}(r)$, we must have $X=Y_{A}$ for some $A \in U \backslash X$. Hence, we have $X \in B_{s}(r)$.

We can note that the base $B_{s}(r)$ is de- fined at the level of single attributes $A \in U$, which shows the necessity that the semantics complies with the definition 3 .

From a data mining point of view, the computation of $B_{s}(r)$ is a crucial step since data accesses are only performed here.

Example 11 Consider the semantics $s_{1}$ and the relation $r$ made of 5 tuples over a set of 6 attributes, given in Table 1.

\begin{tabular}{c|cccccc}
$\mathrm{r}$ & $g_{1}$ & $g_{2}$ & $g_{3}$ & $g_{4}$ & $g_{5}$ & $g_{6}$ \\
\hline$t_{1}$ & 1.7 & 1.5 & 1.2 & -0.3 & 1.4 & 1.6 \\
$t_{2}$ & 1.8 & -0.7 & 1.3 & 0.8 & -0.1 & 1.7 \\
$t_{3}$ & -1.8 & 0.4 & 1.7 & 1.8 & 0.6 & -0.4 \\
$t_{4}$ & -1.7 & -1.4 & 0.9 & 0.5 & -1.8 & -0.2 \\
$t_{5}$ & 0.0 & 1.9 & -1.9 & 1.7 & 1.6 & -0.5
\end{tabular}

Table 1: A running example

The base of the closure system $B_{s}(r)$ is computed as follows:

$$
B_{s}(r)=\bigcup_{t \in r}\{A \in U \mid 1.0 \leq t[A] \leq 2.0\} .
$$

For this relation $r$, we have:

$$
\begin{gathered}
B_{s}(r)= \\
\left\{\left\{g_{1}, g_{2}, g_{3}, g_{5}, g_{6}\right\},\left\{g_{1}, g_{3}, g_{6}\right\},\left\{g_{3}, g_{4}\right\},\{\},\left\{g_{2}, g_{4}, g_{5}\right\}\right\} .
\end{gathered}
$$

For example, a minimal cover of satisfied rules can be computed from $B_{s}(r)$ to give the following rules:

$$
\left\{g_{1} \rightarrow\left\{g_{3}, g_{6}\right\},\left\{g_{1}, g_{4}\right\} \rightarrow g_{2}, g_{2} \rightarrow g_{5},\right.
$$$$
\left.\left\{g_{2}, g_{3}\right\} \rightarrow g_{1}, g_{5} \rightarrow g_{2}, g_{6} \rightarrow g_{1}\right\} \text {. }
$$ 


\section{Related contributions}

To the best of our knowledge, we are not aware of related works in the literature dealing with syntactical characterizations with regard to some inference systems. Nevertheless, related contributions have been done on association rules in data mining $[5,9]$ and functional dependencies in data bases $[20,25,29]$. Rule mining often results in a huge amount of rules and as a consequence, rules turn out to be useless for experts. This is the well-known postprocessing step in a KDD process. To address this problem, different lines of research have been performed. Firstly, rules may be filtered out a priori, based on userdefined templates of rules [23, 8]. Secondly, many quality measures have been developed to select only the most interesting rules $[6,30]$ with regard to these measures. Thirdly, inference rules or inference systems have been proposed to reduce the number of rules given to the experts $[9,15,24,26]$. Most of these works consider new inference systems for association rules by taking into account support and confidence thresholds.

In [3], we proposed three new semantics for gene expression data and show their biological interests. In $[2,4]$, we did a first step toward a formal framework with a first generic definition of a semantics and the notion of a well-formed semantics. In this paper, we go a step beyond by giving a new syntactical characterization of a wellformed semantics, leading to the theorem 1.
We also introduce the existing relationship with the data mining problem of discovery of rules.

\section{Conclusion}

In this paper, we have pointed out that an equivalence does exist between some syntactic restrictions on the natural definition of a given semantics and the fact that this semantics is well-formed, i.e. Armstrong's axioms are sound and complete for this semantics. From a practical point of view, this equivalence allows to prove easily whether or not a given semantics is wellformed.

We have illustrated our proposition on many examples of semantics and have shown the usefulness of our proposition as a tool to ensure that classical reasoning on rules with Armstrong's axioms is indeed possible.

We have also pointed out the relationship between our generic definition of rule satisfaction and the underlying data mining problem.

In the context of our application on gene expression data, this work brings some foundations to build new well-formed semantics with biologists which best fit into their requirements. Moreover, the rule discovery process has to be revisited to avoid a costly (exponential in the number of genes) and useless (too many rules being generated to be validated by biologists) generation of rules. 


\section{Acknowledgment}

We wish to thank the reviewers for their helpful comments and suggestions.

\section{References}

[1] S. Abiteboul, R. Hull, and V. Vianu. Fondements des bases de données. Addison Wesley, 2000.

[2] M. Agier and J.-M. Petit. Notion de sémantiques bien-formées pour les règles. In Actes de la conférence $E G C$, volume 1, pages 19-30, Jan. 2005.

[3] M. Agier, J.-M. Petit, V. Chabaud, C. Pradeyrol, Y.-J. Bignon, and V. Vidal. Vers différents types de règles pour les données d'expression de gènesApplication à des données de tumeurs mammaires. In Actes du Congrès INFORSID'04, Biarritz, pages 351-367, May 2004.

[4] M. Agier, J.-M. Petit, and E. Suzuki. Towards ad-hoc rule semantics for gene expression data. In Proc. of the ISMIS, Saratoga Springs, New-York, USA, pages 494-503. Springer-Verlag, 2005.

[5] R. Agrawal, T. Imielinski, and A. N. Swami. Mining association rules between sets of items in large databases. In Proc. of the ACM SIGMOD, Washington D.C., pages 207-216, 1993.

[6] R. Agrawal and R. Srikant. Fast algorithms for mining association rules in large databases. In Proc. of the $20^{\text {th }}$ VLDB, Santiago de Chile, Chile, pages 487-499, 1994.

[7] W. W. Armstrong. Dependency structures of data base relationships. In Proc. of the IFIP Congress 1974, pages 580-583, 1974.

[8] E. Baralis and G. Psaila. Designing templates for mining association rules. J. Intell. Inf. Syst., 9(1):7-32, 1997.

[9] Y. Bastide, N. Pasquier, R. Taouil, G. Stumme, and L. Lakhal. Mining minimal non-redundant association rules using frequent closed itemsets. In Proc. of the $1^{\text {st }}$ Computational Logic, London, UK, volume 1861, pages $972-$ 986, 2000.

[10] C. Becquet, S. Blachon, B. Jeudy, J.-F. Boulicaut, and O. Gandrillon. Strongassociation-rule mining for large-scale gene-expression data analysis: a case study on human sage data. Genome Biology, 3(12), 2002.

[11] C. Beeri and P. Berstein. Computational problems related to the design of normal form relation schemes. $A C M$ TODS, 4(1):30-59, 1979.

[12] C. Beeri, M. Dowd, R. Fagin, and R. Statman. On the structure of Armstrong relations for functional dependencies. JACM, 31(1):30-46, 1984.

[13] G. Cong, A. K. H. Tung, X. Xu, and F. P. andJiong Yang. Farmer: Finding 
interesting rule groups in microarray datasets. In Proc. of the ACM SIGMOD, pages 143-154, 2004.

[14] C. Creighton and S. Hanash. Mining gene expression databases for association rules. Bioinformatics, 19:79-86, 2003.

[15] L. Cristofor and D. A. Simovici. Generating an informative cover for association rules. In Proc. of the IEEE ICDM, Maebashi City, Japan, pages 597-600, 2002.

[16] J. Demetrovics and V. Thi. Some remarks on generating Armstrong and inferring functional dependencies relation. Acta Cybernetica, 12(2):167-180, 1995.

[17] B. Ganter and R. Wille. Formal Concept Analysis. Springer-Verlag, 1999.

[18] G. Gottlob and L. Libkin. Investigations on Armstrong relations, dependency inference, and excluded functional dependencies. Acta Cybernetica, 9(4):385-402, 1990.

[19] J.-L. Guigues and V. Duquenne. Familles minimales d'implications informatives résultant d'un tableau de données binaires. Math. Sci. Humaines, 24(95):5-18, 1986.

[20] Y. Huhtala, J. Kärkkäinen, P. Porkka, and H. Toivonen. Efficient discovery of functional and approximate dependencies using partitions. In Proc. of the $14^{\text {th }}$ IEEE ICDE, pages $392-401$, 1998.

[21] A. Icev, C. Ruiz, and E. F. Ryder. Distance-enhanced association rules for gene expression. In BIOKDD'03, in conjunction with ACM SIGKDD, Washington, DC, USA, 2003.

[22] J. Kivinen and H. Mannila. Approximate inference of functional dependencies from relations. TCS, 149(1):129149, 1995.

[23] M. Klemettinen, H. Mannila, P. Ronkainen, H. Toivonen, and A. Verkamo. Finding Interesting Rules from Large Sets of Discovered Association Rules. In Proc. of the $3^{t d}$ CIKM, pages 401-407, Dec. 1994.

[24] G. Li and H. Hamilton. Basic association rules. In Proc. of the $4^{\text {th }}$ SIAM ICDM, Lake Buena Vista, Florida, USA, 2004.

[25] S. Lopes, J.-M. Petit, and L. Lakhal. Functional and approximate dependencies mining: Databases and FCA point of view. JETAI, 14(2/3):93-114, 2002.

[26] V. P. Luong. The representative basis for association rules. In Proc. of the IEEE ICDM, pages 639-640, 2001.

[27] D. Maier. Minimum covers in the relational database model. JACM, 27(4):664-674, 1980. 
[28] H. Mannila and K.-J. Räihä. Algorithms for inferring functional dependencies from relations. DKE, 12(1):8399, Feb. 1994.

[29] N. Novelli and R. Cicchetti. Fun: An efficient algorithm for mining functional and embedded dependencies. In Proc. of the ICDT, London, UK, volume 1973 of LNCS, pages 189-203. Springer-Verlag, 2001.

[30] P.-N. Tan, V. Kumar, and J. Srivastava. Selecting the right objective measure for association analysis. Information Systems, 29(4):293-313, Jun. 2004.

[31] M. J. Zaki. Generating non-redundant association rules. In Proceedings of the sixth ACM SIGKDD international conference on Knowledge discovery and data mining, pages 34-43. ACM Press, 2000.

\section{$9 \quad$ Appendix}

Proof of Theorem 1 Let $s \in C$ be a rule semantics. We have first to prove that if $s \in C_{A}$ then $s$ is well-formed and secondly that if $s$ is well-formed then $s \in C_{A}$ or equivalently that if $s \notin C_{A}$ then $s$ is not well-formed.

Lemma 1 Let $s \in C$ be a rule semantics. If $s \in C_{A}$ then the semantics $s$ is wellformed.
Proof 4 (If) Suppose that $s \in C$ i.e. $s$ complies with definition 3 , we have to show that $s$ is well-formed i.e. that Armstrong's axiom system is sound and complete for $s$.

Lemma 2 Armstrong's axiom system is sound for $s$.

Proof 5 Let $F$ be a set of rules over $U$. We need to show that if $F \vdash X \rightarrow Y$ then $F \models_{s} X \rightarrow Y$, i.e. let $r$ be a relation over $U$ such that $r \models_{s} F$, if $F \vdash X \rightarrow Y$ then $r \models_{s} X \rightarrow Y$.

1. (reflexivity) We have to show that if $X \subseteq Y \subseteq U$ then $r \models_{s} Y \rightarrow X$.

Let $X \subseteq Y \subseteq U$ and let $r^{\prime} \subseteq r$ verifying $d_{c}\left(r^{\prime}\right)$ and $\forall A \in Y, \operatorname{Pred}\left(A, r^{\prime}\right)$ is true. Since $X \subseteq Y$, the result follows.

2. (augmentation) We have to show that if $F \vdash X \rightarrow Y$ and $W \subseteq U$, then $r \models_{s} X W \rightarrow Y W$.

Let $r^{\prime} \subseteq r$ verifying $d_{c}\left(r^{\prime}\right)$ and $\forall A \in$ $X \cup W, \operatorname{Pred}\left(A, r^{\prime}\right)$ is true. If $F \vdash$ $X \rightarrow Y$, then we have $\forall B \in Y$, $\operatorname{Pred}\left(B, r^{\prime}\right)$ is true. The result follows.

3. (transitivity) We have to show that if $F \vdash X \rightarrow Y$ and $F \vdash Y \rightarrow Z$ then $r \models{ }_{s} X \rightarrow Z$.

Let $r^{\prime} \subseteq r$ verifying $d_{c}\left(r^{\prime}\right)$ and $\forall A \in X$, $\operatorname{Pred}\left(A, r^{\prime}\right)$ is true. If $F \vdash X \rightarrow Y$ and $F \vdash Y \rightarrow Z$, then $\forall B \in Y, \operatorname{Pred}\left(B, r^{\prime}\right)$ is true and $\forall C \in Z, \operatorname{Pred}\left(C, r^{\prime}\right)$ is true respectively. The result follows.

Note here that the proof is indeed possible thanks to the restriction posed on the 
predicate, satisfied for each single attribute $A \in X$, in definition 3 .

Lemma 3 Armstrong's axiom system is complete for $s$.

Proof 6 We need to show that if $F \models_{s}$ $X \rightarrow Y$ then $F \vdash X \rightarrow Y$ or equivalently, if $F \nvdash X \rightarrow Y$ then $F \not{ }_{s} X \rightarrow Y$. As a consequence, assuming that $F \nvdash X \rightarrow Y$, it is enough to give a counter-example relation $r$ such that $r \models_{s} F$ but $r \not \models_{s} X \rightarrow Y$.

Note here that to exhibit the counterexample $r$, we do not have to explicitly give the data instance. Using the constraint $d_{c}(r)$, we just have to build a relation verifying this constraint, whatever the real values are.

Let $r$ be a relation verifying $d_{c}(r)$ such that $\forall A \in X^{+}, \operatorname{Pred}(A, r)$ is true and $\forall B \in$ $U \backslash X^{+}, \operatorname{Pred}(B, r)$ is false.

Clearly, such a relation always exists whenever Pred is not logically equivalent to true (or false) (cf section 3.1).

Let us recall that $X^{+}=\{A \in U \mid F \vdash$ $X \rightarrow A\}$.

One can note, by the construction of $r$, that $r \not \nvdash_{s} V \rightarrow W$ if and only if $V \in X^{+}$and $\exists A \in W$ such that $A \in U \backslash X^{+}$. Otherwise, $r \models_{s} V \rightarrow W$.

Firstly, we have to show that $r \models_{s} F$. We suppose the contrary that $r \nvdash_{s} F$ and thus, $\exists V \rightarrow W \in F$ such that $r \not \nvdash_{s} V \rightarrow$ $W$. It follows by the construction of $r$ that $V \subseteq X^{+}$and $\exists A \in W$ such that $A \in U \backslash$ $X^{+}$. Since $V \in X^{+}$, we have $F \vdash X \rightarrow$ $V$ and since $F \vdash V \rightarrow W$, we have $F \vdash$
$V \rightarrow A$. Thus, by the transitivity rule, $F \vdash$ $X \rightarrow A$ and thus $A \in X^{+}$. This leads to a contradiction since $A \in W$, and thus $r \models_{s}$ $F$.

Secondly, we have to show that $r \nvdash_{s}$ $X \rightarrow Y$. We suppose the contrary that $r \models{ }_{s} X \rightarrow Y$. It follows by the construction of $r$ that $Y \subseteq X^{+}$and thus $F \vdash X \rightarrow Y$. It leads to a contradiction since $F \forall X \rightarrow Y$ was assumed, and thus $r \not \nvdash_{s} X \rightarrow Y$.

The second part of the proof is certainly the more surprising one since it tells us that any well-formed semantics has to comply with definition 3 :

Lemma 4 Let $s \in C$ be a rule semantics. If the semantics $s$ is well-formed, then $s \in$ $C_{A}$.

Proof 7 (Only if) We have to show that if $s$ is well-formed, then $s \in C_{A}$ or equivalently, if $s \notin C_{A}$ (i.e. $s$ does not comply with definition 3 ) then $s$ is not well-formed.

Suppose that $s$ does not comply with definition 3, two cases are possible: Either the two predicates are different or they are equivalent but it does not exist an equivalent predicate which can be formulated as a condition on each attribute.

Let us consider the first case, i.e. Pred $_{1}$ and Pred $_{2}$ are not equivalent: In that case, it does exist a relation $r$ and a subset of attributes $Y \subseteq U$ such that $\left\{r^{\prime} \subseteq r \mid r^{\prime}\right.$ verifying $d_{c}\left(r^{\prime}\right)$ and $\operatorname{Pred}_{1}\left(Y, r^{\prime}\right)$ is true $\} \neq$ $\left\{r^{\prime} \subseteq r \mid r^{\prime}\right.$ verifying $d_{c}\left(r^{\prime}\right)$ and $\operatorname{Pred}_{2}\left(Y, r^{\prime}\right)$ is true\}. 
Two cases are thus possible:

- $\exists r^{\prime} \subseteq r$ verifying $d_{c}\left(r^{\prime}\right)$ such that $\operatorname{Pred}_{1}\left(Y, r^{\prime}\right)$ is true and $\operatorname{Pred}_{2}\left(Y, r^{\prime}\right)$ is false:

Let us assume that $s$ is well-formed. By reflexivity, we have $\forall X \subseteq Y, r \models$ $Y \rightarrow X$ and thus $r \models Y \rightarrow Y$ i.e. $\forall r^{\prime} \subseteq r$ verifying $d_{c}\left(r^{\prime}\right)$, if $\operatorname{Pred}_{1}\left(Y, r^{\prime}\right)$ is true then $\operatorname{Pred}_{2}\left(Y, r^{\prime}\right)$ is true which is a contradiction.

- $\exists r^{\prime} \subseteq r$ verifying $d_{c}\left(r^{\prime}\right)$ such that $\operatorname{Pred}_{1}\left(Y, r^{\prime}\right)$ is false and $\operatorname{Pred}_{2}\left(Y, r^{\prime}\right)$ is true:

Without loss of generality, let us assume there exists $X \in U \backslash Y$ and $Z \in$ $U \backslash Y$ such that $\operatorname{Pred}_{1}\left(X, r^{\prime}\right)$ is true and $\operatorname{Pred}_{2}\left(Z, r^{\prime}\right)$ is false, as depicted in Table 2. Thus, we have $r^{\prime} \models X \rightarrow Y$ and $r^{\prime} \models Y \rightarrow Z$.

\begin{tabular}{c|c|c|c|c}
$r$ & $X$ & $Y$ & $Z$ & $\ldots$ \\
\hline$\ldots$ & $\ldots$ & $\ldots$ & $\ldots$ & $\ldots$ \\
$r^{\prime}\{$ & $P_{1}$ true & $P_{1}$ false & & \\
$\ldots$ & & $P_{2}$ true & $P_{2}$ false & $\ldots$ \\
$\ldots$ & $\ldots$ & $\ldots$ & $\ldots$
\end{tabular}

Table 2: Example

Assume now that $s$ is well-formed. By transitivity, we should have $r^{\prime} \models X \rightarrow$ $Z$, which is false and leads to a contradiction.

Finally, we have shown that if Pred $_{1}$ and Pred $_{2}$ are not equivalent, then $s$ is not-wellformed.
Now, let us consider the second case, i.e. Pred $_{1} \equiv$ Pred $_{2}$ but they are not equivalent to the predicate $\operatorname{Pred}^{\prime}(Y)=[\forall A \in$ $\left.Y, \operatorname{Pred}_{1}(A)\right]$ : In that case, it does exist a relation $r$ and a subset of attributes $Y \subseteq U$ such that $\left\{r^{\prime} \subseteq r \mid r^{\prime}\right.$ verifying $d_{c}\left(r^{\prime}\right)$ and $\operatorname{Pred}_{1}\left(Y, r^{\prime}\right)$ is true $\} \neq\left\{r^{\prime} \subseteq r \mid r^{\prime}\right.$ verifying $d_{c}\left(r^{\prime}\right)$ and $\forall A \in Y, \operatorname{Pred}_{1}\left(A, r^{\prime}\right)$ is true $\}$. We can show that reflexivity and transitivity axioms are not sound in $r^{\prime}$. The proof is similar to the previous one and is omitted. 